\title{
The combination of resonance Raman spectroscopy, optical tweezers and microfluidic systems applied to the study of various heme-containing single cells
}

\author{
K. Ramser ${ }^{\mathrm{a}, *}$, W. Wenseleers ${ }^{\mathrm{b}}$, S. Dewilde ${ }^{\mathrm{c}}, \mathrm{S}$. Van Doorslaer ${ }^{\mathrm{b}}$ and L. Moens ${ }^{\mathrm{c}}$ \\ a Department of Computer Science and Electrical Engineering, Luleå University of Technology, Luleå, \\ Sweden \\ ${ }^{\mathrm{b}}$ Department of Physics, University of Antwerp, Antwerp, Belgium \\ ${ }^{\mathrm{c}}$ Department of Biomedical Sciences, University of Antwerp, Antwerp, Belgium
}

\begin{abstract}
Several recent studies on the function of neuroglobin $(\mathrm{Ngb})$, a hemoprotein predominantly expressed in the brain, point toward a neuro-protective role during hypoxic-ischemic injuries. The exact mechanism by which Ngb protects the cell against $\mathrm{H}_{2} \mathrm{O}_{2}$-induced cell death remains to be elucidated. Hence, new tools need to be developed in order to study the protein in vivo or under physiological conditions. In this summary of our work, we demonstrate how resonance Raman spectroscopy, optical tweezers and microfluidic systems were combined to mimic in vivo conditions in an in vitro milieu. The setup has been tested on several globin-containing cells: hemoglobin $(\mathrm{Hb})$ within single red blood cells (RBCs), a nerve globin present in the nerve cord of the annelid Aphrodite aculeata (A. aculeata), and wild-type (wt) human neuroglobin (NGB) overexpressed in Escherichia coli (E. coli) bacteria. The feasibility of the setup regarding sensitivity and photo-induced effects and the results regarding the oxygen uptake and release will be discussed and compared for each system. The summary of the results show that the method is promising and the setup will be developed further to monitor the dependence of the neuronal action potential on nerve globins.
\end{abstract}

Keywords: Neuroglobin, hemoglobin, nerve myoglobin, resonance Raman spectroscopy, optical tweezers, microfluidic system

\section{Introduction}

Ligand-binding globins are found throughout all kingdoms of nature. Various functions are identified, such as oxygen delivery and storage and enzymatic activities. Newly revealed paths for ligand binding in mammalian globins show that these heme proteins are much more versatile in their structure/function relationship than so far thought [1]. The discovery of a new mammalian globin [2], predominantly expressed in the brain and therefore termed neuroglobin $(\mathrm{Ngb})$, stimulated the heme research even more and special interest was drawn towards nerve globins. In the past, the function of invertebrate nerve hemoglobins as oxygen stores that extend neural excitability under hypoxia has been convincingly demonstrated [3]. The physiological role of vertebrate Ngbs on the other hand, is less well understood

\footnotetext{
${ }^{*}$ Corresponding author: K. Ramser, Department of Computer Science and Electrical Engineering, Luleå University of Technology, SE-971 87 Luleå, Sweden. Tel.: +46 920 491648; Fax: +46 920 493111; E-mail: kerstin.ramser@ltu.se.
} 
[4]. Several recent studies point toward a neuro-protective role during hypoxic-ischemic injuries: Ngb is up-regulated under oxygen deprivation and protects the brain from experimental stroke, which has been shown both in vitro and in vivo [5,6]. Human neuroglobin (NGB) is an efficient scavenger of reactive oxygen species $[7,8]$, and the potential of the protein to function as an enzyme or substrate has been demonstrated [9]. Peroni et al. applied intracellular delivery of Ngb using the HIV-1 TAT protein transduction domain, but in this study the protection against oxygen and glucose deprivation failed [10]. The authors suggest that the reason for the failure might have been the applied experimental model for oxygen glucose deprivation, or alternatively, that the intracellular delivery of Ngb by TAT/CPP does not have a beneficial effect in the treatment of ischemic pathology.

It is important to note, that despite of the majority of positive findings listed above, the exact mechanism by which $\mathrm{Ngb}$ protects the cell against $\mathrm{H}_{2} \mathrm{O}_{2}$-induced cell death remains to be elucidated. In contrast to metMb and metHb, metNGB does not form the cytoxic, strong oxidizing ferryl derivative, which results in the formation of secondary radicals [8]. This feature of NGB may be related to the hexacoordinated heme, hence it is likely that NGB plays a key role in the cellular defence against oxidative stress. Neutralization of $\mathrm{O}_{2}$ or $\mathrm{NO}$ can occur with or without the modification of the oxidation state of the heme iron. If the heme iron becomes oxidized in the process, there is a need for reduction of the iron in order to restore the ligand binding capacity. Although this reduction may originally have been achieved by small molecules such as $\mathrm{NAD}(\mathrm{P}) \mathrm{H}$, it is likely that other enzymatic systems acquired this function later on through evolution [9]. The experiments performed by Trandafir et al. demonstrate that the disulfide bond in NGB is enzymatically reduced directly by Thioredoxin reductase (TrxR) [9]. The $\operatorname{Trx} / \operatorname{TrxR}$ system is present in all animal tissue and has an important function in nerve cell metabolism in the reduction of protein disulfides and as ROS scavengers, especially in regions with high energy demands. The authors suggest that TrxR and NGB belong to the defence system of the nerve cell against hypoxia and the oxidative damage occurring by reoxygenation. However, this suggestion demands that the neuroglobins are able to form disulfide bridges, which so far only has been shown in vitro [11]. There is a clear demand to study functional cells under varying physiological conditions. Even though first successful in vivo experiments performed on mice have been reported [6], further tools need to be developed to study functional heme-containing cells under varying physiological conditions in order to elucidate the mechanisms of cell protection against oxidative stress.

The summary of our work presented here has been dedicated to develop tools to study the real time response of single heme-protein-containing cells to environmental changes. Resonance Raman spectroscopy was combined with optical tweezers and microfluidic systems. Resonance Raman spectroscopy is an excellent technique to study heme proteins $[12,13]$ and was used here to register the oxygenation cycle of globins in cells triggered either by the addition of sodium dithionite or by fluctuating aerobic or anaerobic conditions. By applying appropriate laser excitation wavelengths, the resonance effect in the heme group gets strong enough to enable single cell studies without interference by surrounding media or other components of the sample [14]. Microfluidic systems were used to control the environment of the cells. They consist of miniature structures of channels and reservoirs that fit onto a cover glass and give control over the environment in a confined space, mimicking in vivo conditions in an in vitro milieu [15]. The immobilization of the cells was achieved by optical tweezers, i.e. the cells are trapped in a strongly focused near-infrared laser beam. Optical tweezers have become important tools for many biological applications $[16,17]$.

Our studies on heme protein containing cells started out with investigations of single red blood cells (RBCs) [18]. The further development of the technique was encouraged by the discovery of Ngb and 
the aspiration to investigate the protein in functional cells under physiological-like conditions. The feasibility of our approach was tested on Escherichia coli (E. coli) bacteria overexpressing wild-type (wt) NGB [19]. The studies carried out under fluctuating aerobic and anaerobic conditions showed that the reversible oxygenation-deoxygenation can be monitored over a long period of time. Further, the experiment helped to verify the presence of an enzymatic system in the bacteria reducing the ferric form of NGB [9].

Due to parallels of Ngb with nerve globins found in several invertebrates, the nerve cord of the annelid Aphrodite aculeata (A. aculeata) was used as a model system [20]. This study showed that the fast reaction to varying $\mathrm{O}_{2}$ concentrations supports an oxygen-carrying and/or -storing function of the nerve globin in single cells. In addition, by combining resonance Raman and absorption spectroscopy, the physiological response could be distinguished from light-induced effects. In this article we will summarize and compare the results of our studies and report about the further development of the technique that will enable to study the dependence of the action potential of neuronal cells and nerve cords upon heme proteins.

\section{Materials and methods}

The development and improvement of the setup will be presented in an overview, for more information please read Refs [19-21]. The starting point of the design of the experimental setup has in all studies been the Raman spectrometer. Two possible microscope configurations were tested related to the initial configuration of the used Raman spectrometers. If the spectrometer was equipped with an upright microscope, a double microscope configuration was chosen to enable the integration of the optical trap together with the microfluidic system [21], meaning that the optical path for the optical tweezers and the Raman spectrometer were separated. This had the advantage, that different microscope objectives could be used for optical trapping and for the Raman measurements. If no microscope was initially connected to the Raman spectrometer an inverted microscope was built and the same objective was used for optical trapping and Raman measurements. This had the benefit, that the optical system was less complicated [19,20]. Optical trapping was either achieved with a diode laser $(830 \mathrm{~nm})$ or a Nd: $\mathrm{YVO}_{4}$ laser (1064 nm). Raman excitation was generated by an Ar-ion (514 nm), a Kr-ion laser (413 nm) or an ArKr-ion laser (413 nm, $568 \mathrm{~nm})$, respectively.

In the first studies, the microfluidic system was produced by e-beam lithography in an advanced clean room. The final product consisted of a system of channels $(\varnothing=20 \mu \mathrm{m})$ and reservoirs $(\varnothing=2 \mathrm{~mm})$. The flow cell consisted of three different reservoirs: an inlet, a measurement reservoir where the RBCs were studied, and an outlet. By applying a voltage over the in- and outlet an electro-osmotic flow of physiological buffer could be generated. In this case, the oxygenation cycle was triggered by adding sodium dithionite [21]. Since this was a rather crude way to prompt the oxygen release, a gas tight flow cell where the aerobic conditions could be controlled was designed; i.e. a flow through a gas tight Plexiglas cell ( $\varnothing=5 \mathrm{~mm}, h=0.5 \mathrm{~mm}$ ) was generated by a continuous pump that was connected to two vessels containing buffer either purged with $\mathrm{O}_{2}$ or $\mathrm{N}_{2}$, respectively. By switching between the two vessels the flow cell was either flushed with anaerobic or aerobic buffer $[19,20]$.

When red blood cells were studied, a drop of blood $(1 \mu \mathrm{l})$ from a healthy volunteer was diluted in $200 \mu \mathrm{l}$ of Hepes buffer [21]. The nerve cord was dissected and isolated from of the annelid A. aculeata and was put directly into the flow cell filled with fresh seawater [20]. In the experiment where E. coli bacteria overexpressing Ngb were studied [19], the cells were grown according to Dewilde et al. [22]. 
The cell suspension was washed in buffer (5-mM glucose, $25 \mathrm{mM}$ Tris $\mathrm{pH} 8$, and 10-mM EDTA) and diluted $10^{6}$ times for the experiments presented here.

\section{Overview of results}

The heme iron atom forms the heart of the ligand-binding site of the heme protein. In all globins, the heme iron is attached to the protein via the binding of the proximal histidine residue. The remaining axial coordination site on the distal side is available for exogeneous or endogeneous ligand binding. Resonance Raman spectroscopy is an excellent tool to investigate the oxidation and spin state of the heme. The vibrational modes of the chromophoric heme group are selectively resonance enhanced and information about the conformational changes and/or changes in the spin and oxidation state of the iron can be gained. Moreover, if the resonance effect is high enough, it is possible to look at a molecule of interest without the interference by the surrounding media or other components of the sample and hence, it is in principle possible to perform in vivo studies of single cells. Typical resonance Raman spectra of the deoxy and the oxy state of $\mathrm{Hb}$ from a single trapped red blood cell (uppermost two spectra) and of ferrous and ferric NGB over-expressed in E. coli bacteria are shown in Fig. 1.

The different states can be distinguished easily on the basis of the four spin-state and oxidation markers given in Table 1, except for the difference between an oxy form and a bis-histidine coordinated ferric form of a globin. Since the oxy form is a His- $\mathrm{Fe}^{3+}-\mathrm{O}_{2}{ }^{-}$form rather than a $\mathrm{His}-\mathrm{Fe}^{2+}-\mathrm{O}_{2}$ form, it is clear that the oxidation markers (both Fe(III)) and spin-state markers (both low-spin states) will not differentiate the two forms. In order to distinguish between the two forms, observation of other markers, such as the $\nu_{\mathrm{Fe}-\mathrm{OO}}$, is needed, but in most practical cases, the resolution of the single-cell Raman spectra are too low. In this case, a combination with a UV/Vis set-up can be very helpful [20]. Note that the uppermost two spectra in Fig. 1 are excited at $514 \mathrm{~nm}$, whereas the two spectra at the bottom are enhanced at $413 \mathrm{~nm}$ laser wavelength. As a consequence the marker signals are enhanced differently. This is due to the fact that the $413 \mathrm{~nm}$ laser line lies close to the peak of the Soret band, whereas the $514 \mathrm{~nm}$ excitation line is closer to the $\beta$-band stemming from the $Q$ electronic transition of the $\pi^{*}$ electrons of the porphyrin ring [23].

Laser excitation in the absorption bands results in an excellent resonance enhancement, however, the drawback is that unwanted photo-induced chemistry can occur. Hence, a lot of our work has been dedicated to investigate the impact of different laser wavelengths and laser powers. In the first set of experiments RBCs were used as a model system [24]. Using an excitation wavelength of $514 \mathrm{~nm}$, the metHb state was reached simultaneously with the appearance of a fluorescent background after a photon dose of $\sim 1.16 \times 10^{19}$. In a further study, we examined whether the $568 \mathrm{~nm}$ laser line would be more suitable for resonance Raman enhancement of RBCs. As expected from the literature [25,26], a great improvement was observed and measurements could be carried out for long times without any noticeable photo-induced degradation. Consequently, this wavelength was chosen when the nerve cord of $A$. aculeata was examined. To our surprise, this resulted in a strong orange-red fluorescence that was visible with the naked eye. The fluorescence spectrum of the exposed nerve cord showed that the data corresponded to the fluorescence spectrum of biliverdin, a decay product of the heme. Hence, the porphyrin ring is broken up by laser illumination with biliverdin as a photoproduct $[27,28]$. The process involves singlet oxygen as the main cytotoxic agent responsible for the photodynamic effect. It is worthwhile to notice that less photo-induced chemistry was observed in the nerve cord of A. aculeata when $413 \mathrm{~nm}$ was applied as an excitation wavelength. However, care has to be taken even at moderate powers in 


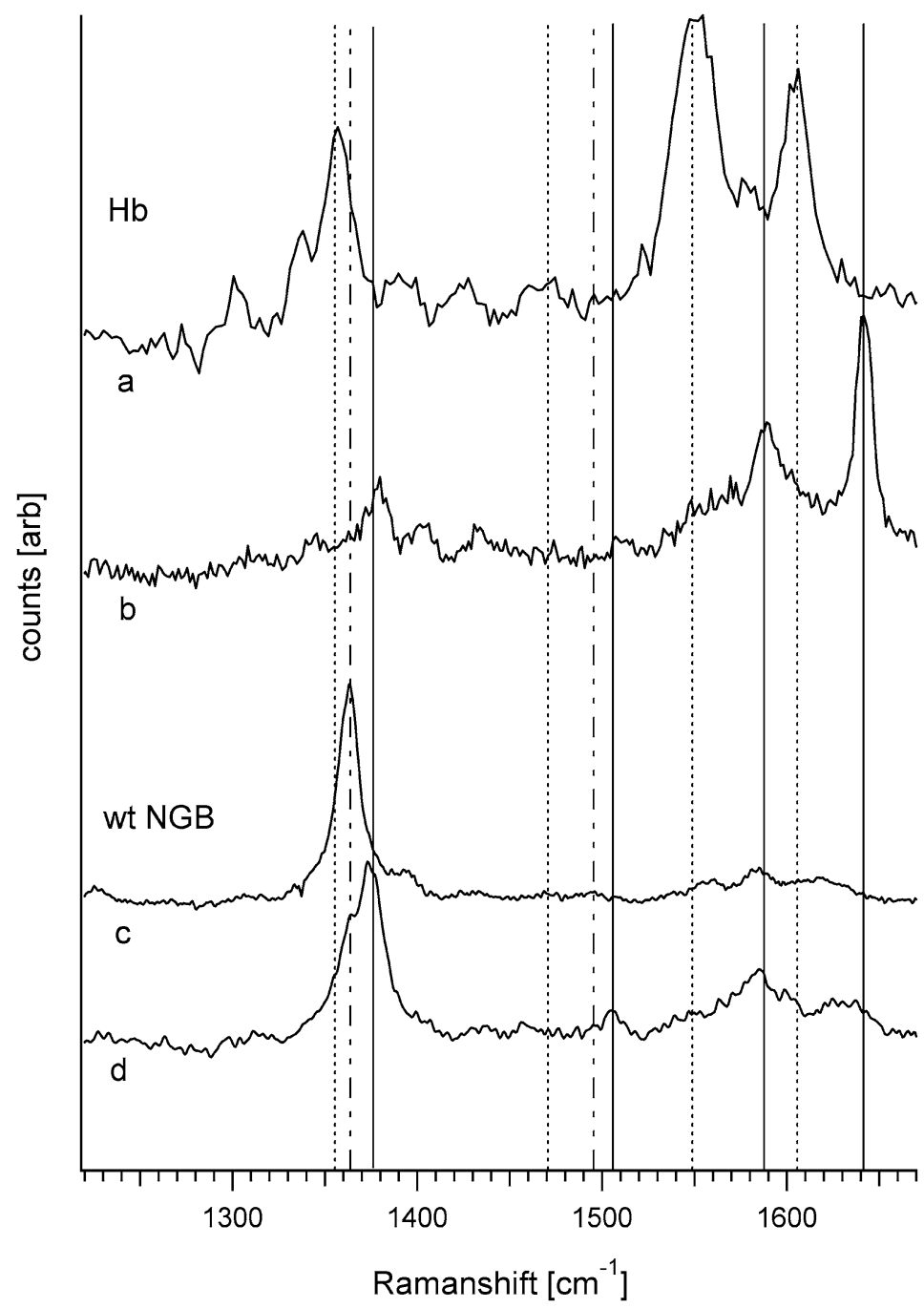

Fig. 1. The resonance Raman spectra of two forms of human $\mathrm{Hb}$ are shown in the two uppermost spectra. The laser excitation line is $514 \mathrm{~nm}$. (a) Resonance Raman spectrum from the penta-coordinated $\mathrm{Fe}^{2+}$ state, also referred to as the deoxy ferrous form. (b) Resonance Raman spectrum from the $\mathrm{O}_{2}$-bound form (the oxy form) of $\mathrm{Hb}$. The bottom two resonance Raman spectra show two forms of wt NGB. The laser excitation was done at $413 \mathrm{~nm}$. (c) The spectrum from the bis-histidine coordinated ferrous state of wt NGB. (d) The resonance Raman spectrum of bis-histidine coordinated ferric state of wt NGB. All shown spectra stem from single-cell experiments. The oxidation and spin-state markers given in Table 1 are indicated in the figure for the deoxy ferrous form (dashed lines), the bis-histidine coordinated ferrous form (dashed-dotted lines) and the identical markers of the oxy form and the bis-histidine coordinated ferric form (full line).

order to avoid all possible photo-induced effects. For instance, upon excitation it is possible to knock off oxygen from the heme group at a certain laser power, which may incorrectly be interpreted as a step in the in vivo oxygenation cycle [20].

Since the cells are immobilized by optical tweezers, possible effects induced by the trapping laser have to be investigated. We used both $830 \mathrm{~nm}$ and $1064 \mathrm{~nm}$ laser wavelengths in the near infra-red region where the absorption in water is low. This eliminates possible heating to a certain degree. We observed that the red blood cells were affected by the $1064 \mathrm{~nm}$ but not by the $830 \mathrm{~nm}$ laser light, whereby the 
Table 1

Position of different oxidation and spin-state markers of heme proteins taken from [13,14]

\begin{tabular}{|c|c|c|c|c|}
\hline \multirow[t]{2}{*}{ State } & \multicolumn{4}{|c|}{ Raman shift $\left(\mathrm{cm}^{-1}\right)$} \\
\hline & $\nu_{4}$ & $\nu_{3}$ & $\nu_{2}$ & $\nu_{10}$ \\
\hline Penta-coordinated $\mathrm{Fe}^{2+}$ form (deoxy ferrous form, His- $\mathrm{Fe}^{2+}$ ) & 1356 & 1470 & 1552 & 1607 \\
\hline$O x y$ form (His- $\left.-\mathrm{Fe}^{3+}-\mathrm{O}_{2}{ }^{-}\right)$ & 1374 & 1505 & 1586 & 1640 \\
\hline Aquomet form $\left(\mathrm{His}-\mathrm{Fe}^{3+}-\mathrm{H}_{2} \mathrm{O}\right)$ & 1373 & 1483 & 1563 & 1608 \\
\hline Bis-histidine coordinated ferric form $\left(\mathrm{His}-\mathrm{Fe}^{3+}-\mathrm{His}\right)$ & 1374 & 1505 & 1586 & 1640 \\
\hline Bis-histidine coordinated ferrous form (His- $\left.\mathrm{Fe}^{2+}-\mathrm{His}\right)$ & 1361 & 1493 & 1586 & \\
\hline
\end{tabular}

negative impact of the $1064 \mathrm{~nm}$ light could be reduced by applying a constant flow of cold fresh buffer.

After a careful choice of the appropriate laser wavelengths and powers, the oxygen uptake and release was studied for RBCs, the nerve cord of A. aculeata and wt NGB over-expressed in E. coli bacteria (Fig. 2). The top graph shows the oxygen release and uptake from a single trapped RBC by plotting the ratio of the intensity of the $\nu_{10}$ band at $1607 \mathrm{~cm}^{-1}$ (deoxy) and $1640 \mathrm{~cm}^{-1}$ (oxy) against time (min). The full experimental details can be found in [21] and will only briefly be described here. A high value of the $\nu_{10}$-intensity ratio indicates that no oxygen is bound to the heme. The excitation wavelength was $514 \mathrm{~nm}$ and the trapping wavelength was $830 \mathrm{~nm}$. An electro-osmotic flow of cold fresh buffer was generated by applying a voltage over the electrodes. Initially, $\mathrm{Hb}$ within the single trapped cell is in an mixed state, some of the $\mathrm{Hb}$ is bound to $\mathrm{O}_{2}$ and some is in the deoxy form. By flushing the sample with cold fresh buffer all of the $\mathrm{Hb}$ binds to $\mathrm{O}_{2}$. Addition of sodium dithionite in the inlet reservoir (after the 4th spectrum) triggered the release of oxygen. By flushing the sample with cold fresh $\mathrm{O}_{2}$ containing buffer the trapped RBC turned into a mixed oxy-deoxy state after approximately 13 min before reaching the metHb state after another $40 \mathrm{~s}$.

The middle graph in Fig. 2 shows the oxygen uptake and release of the nerve cord of A. aculeata [20]. Since the globin within the nerve cord was extremely susceptible to photo-induced effects, the oxygenation cycle was monitored with resonance Raman spectroscopy and absorption spectroscopy simultaneously. Due to the macroscopic size of the dissected nerve cord, no optical trap was applied in this experiment. The absorption spectrum was used to ensure that the oxygenation cycle was true and not photo-induced and also to facilitate the distinction between the oxyHb and the metHb state. For the Raman spectra (excited with $413 \mathrm{~nm}$ ), the ratio between $1361 \mathrm{~cm}^{-1}$ (deoxy) and $1374 \mathrm{~cm}^{-1}$ (oxy) of the $\nu_{4}$ band is plotted against time. For the absorption spectrum, the time dependence of the ratio between $568 \mathrm{~nm}$ (deoxy) and $540 \mathrm{~nm}$ (oxy) is shown.

The bottom graph in Fig. 2 shows the oxygenation cycle of wt NGB over-expressed in E. coli bacteria [19]. About 6 bacteria were trapped for the experiment in order to give a good signal-to-noise ratio. In this case, wt NGB was initially in a ferrous state to start with and even though the cells were flushed heavily with oxygen rich buffer, the partly oxygenated state strived back to the reduced state. The time span measured for the trapped cells to go from a deoxy state to a mixture between the deoxy and oxy form was around $2 \mathrm{~min}$. Approximately $20 \mathrm{~min}$ after turning of the flow the reduced state was reached again. It is surprising to see that the oxygenated wt NGB returns to a completely reduced state even under oxygen rich conditions [22], given the fact that NGB has a very high auto-oxidation rate in vitro. 


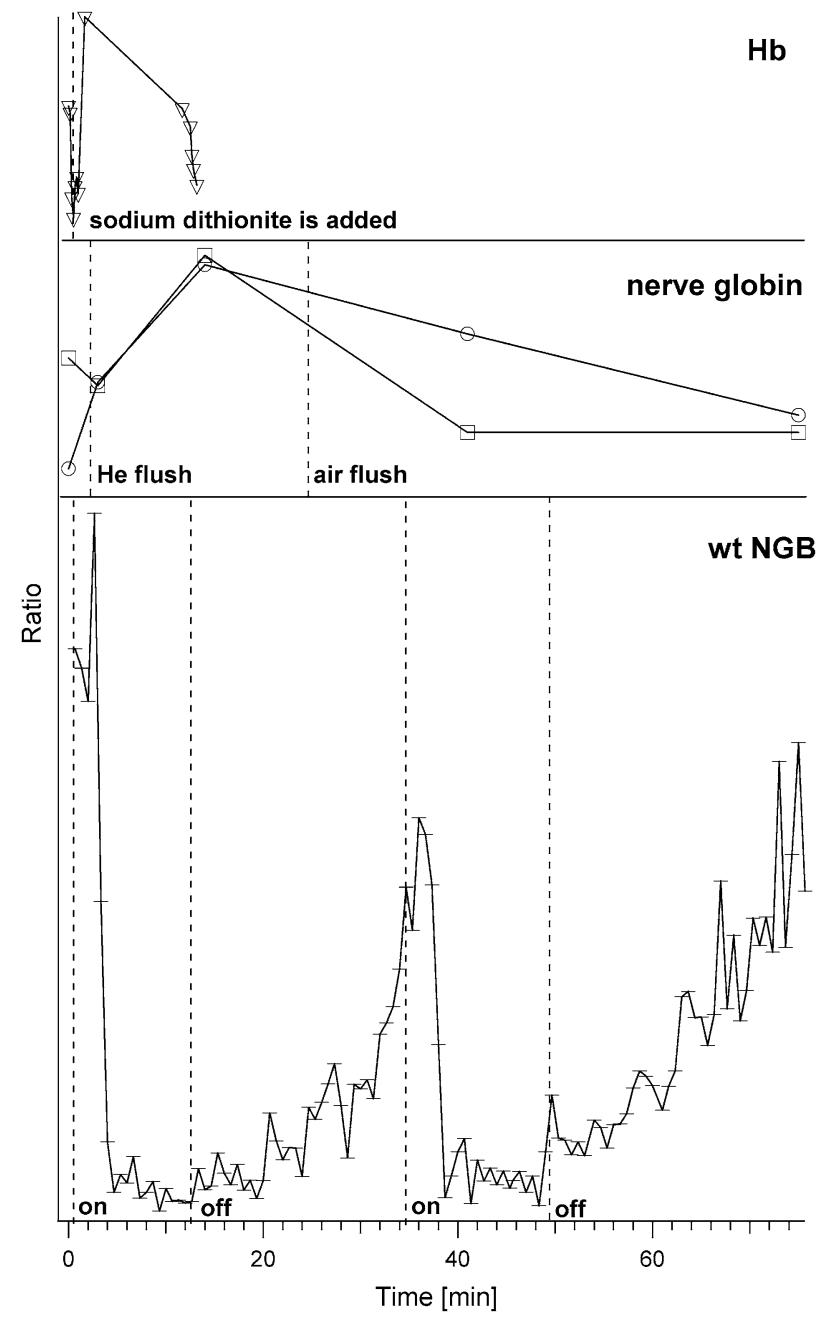

Fig. 2. Top: the oxygen uptake and release of human $\mathrm{Hb}$ in RBCs. The ratio of the $\nu_{10}$ band at 1607 (deoxy) and $1640 \mathrm{~cm}^{-1}$ (oxy) is plotted versus time (min). The Raman laser excitation line was $514 \mathrm{~nm}$, the power prior to the microscope objective was $0.9 \mathrm{~mW}$, the integration time was $10 \mathrm{~s}$. The trapping laser $(1064 \mathrm{~nm})$ was set to $90 \mathrm{~mW}$. A flow of cold buffer was constantly on. After $40 \mathrm{~s}$ (see dotted line) sodium dithionite was added to the buffer. Middle: the oxygen uptake and release of the nerve globin in the nerve cord of A. aculeata. The ratio of the $\nu_{4}$ band at 1361 and $1374 \mathrm{~cm}^{-1}$ in the resonance Raman spectra is shown as a function of time excitation wavelength is $413 \mathrm{~nm}$, integration time is $20 \mathrm{~s}$. Laser power is $0.07 \mathrm{~mW}$ prior to the microscope objective. Furthermore, the time dependence of the ratio between the absorption peaks at 568 and $540 \mathrm{~nm}$ is plotted. The dotted vertical lines indicate when the flush was changed from anaerobic to aerobic seawater. Bottom: the oxygen uptake and release of wt NGB in E. coli bacteria: the ratio of the $\nu_{4}$ band at 1361 and $1374 \mathrm{~cm}^{-1}$ in the resonance Raman spectra is shown as a function of time. An excitation line of $413 \mathrm{~nm}, 0.43 \mathrm{~mW}$ laser power and an integration time $40 \mathrm{~s} \mathrm{per} \mathrm{spectrum} \mathrm{was} \mathrm{taken.} \mathrm{The}$ trapping laser $(830 \mathrm{~nm})$ power was $80 \mathrm{~mW}$. The dotted lines indicate the change of aerobic conditions.

\section{Discussion and future outlook}

The ongoing development of the setup combining resonance Raman spectroscopy with optical tweezers and microfluidic systems in our lab has given us the opportunity to study various heme-containing functional cells and model systems. The experimental conditions were improved in order to mimic in vivo conditions in an in vitro milieu [21]. In the first experiments the oxygen release in RBCs was trig- 
gered by addition of sodium dithionite (Fig. 2(top)). This cannot be considered physiological. In fact, the $\mathrm{Hb}$ within the cells turned into the metHb state after one cycle that lasted for approximately 13 min. However, human $\mathrm{Hb}$ and RBCs are among the most well studied biological systems there are, hence they served as an excellent starting point for the development of the technique. Also the high concentration of $\mathrm{Hb}$ within the RBC $\left(300 \times 10^{-13} \mathrm{~g} \mathrm{Hb}\right)$ and the considerable size of the blood cells $\left(100 \mu \mathrm{m}^{3}\right)$ facilitated this development. When the first experiments on E. coli bacterium overexpressing wt NGB were performed, the sensitivity and resolution of the setup needed to be improved, since $E$. coli bacteria are about 100 times smaller than RBCs and the protein concentration is 10 times less [19]. By gaining control over photo-induced effects and by improving the set up by adding a gas tight flow cell that allows for a good control of the (an)aerobic conditions, the observation time could be prolonged significantly, as can be seen from Fig. 2(bottom). Furthermore, the same cell could be observed for at least 75 min without noticeable protein degradation and the oxygen uptake and release could be monitored reversibly. This observation supported the in vitro UV/Vis data that showed that ferric NGB can be easily reduced in aerobic conditions by a non specific reduction system present in the cell, despite of the high auto-oxidation rate of NGB [9]. It is also noteworthy, that different globins can show very different susceptibilities towards photo-induced effects. The nerve globin within the nerve cord of A. aculeata showed to be very sensitive towards the excitation wavelength and laser power chosen [20]. Most surprising was the fact that the photo-induced damage could be reduced with laser light of $413 \mathrm{~nm}$ as opposed to the less energetic laser excitations with longer wavelengths, which in most cases are favourable for frail biological systems [25,26]. The opposite is true for Hb within RBCs: photo damage could be reduced significantly when the excitation wavelength was changed from $514 \mathrm{~nm}$ to $568 \mathrm{~nm}$. For wt NGB in the $E$. coli bacteria no photo-damage was registered.

Future experiments will be directed towards measuring the dependence of the nerve globins on neuronal activity. The goal is to create a measurement cell where aerobic and other physiological conditions such as $\mathrm{pH}$ and temperature can be controlled at the same time as the action potential can be monitored (Fig. 3). This involves micro-manipulation abilities in a gas tight milieu. Due to its high nerve

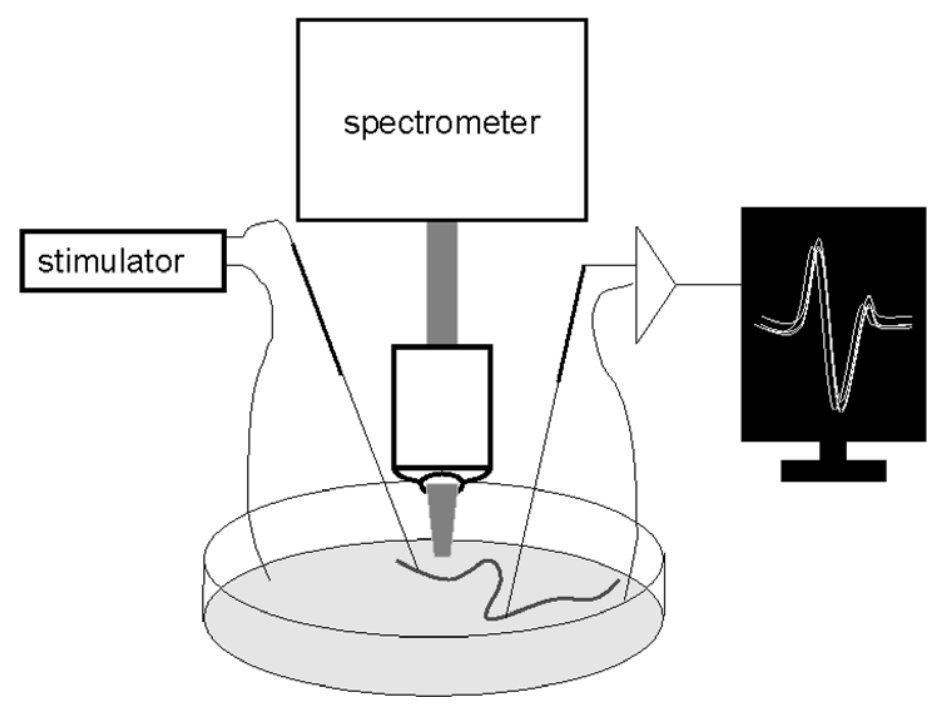

Fig. 3. Schematic representation of the future flow cell. Micromanipulation possibilities will be enabled while the aerobic conditions can be controlled. The action potential will be measured simultaneously with the oxygenation state of nerve globin. In the scheme a nerve cord of $A$. aculeata is depicted. 
globin concentration, the nerve cord of A. aculeata will be chosen as a model system. In the future, the experiments can then be extended to other neuronal cells and systems.

\section{Acknowledgements}

This work was supported by the European Commission 6th framework programme through the project ATOM-3D (Contract No. 508952), and from the European Science Foundation EUROCORES Progamme, SPANAS, through funds from the Swedish Research Council and from the European Commission Sixth Framework Programme. Further acknowledged is the Fund for Scientific ResearchFlanders (FWO) for support through the Grant G.0468.03 and the European Commission project (Contract No. QLG3-CT-2002-01548 to L.M). S.D. and W.W. are postdoctoral fellows of the FWO. For technical assistance we would like to thank Heinrich Riedl and Paul Casteels.

\section{References}

[1] M. Brunori, Trends in Biochemical Sciences 26 (2001), 209-210.

[2] T. Burmester, B. Welch, S. Reinhardt and T. Hankeln, Nature 407 (2000), 520-523.

[3] D.W. Kraus and J.M. Colacino, Science 232 (1986), 90-92.

[4] C. Hundahl, A. Fago, S. Dewilde, L. Moens, T. Hankeln, T. Burmester and R.E. Weber, FEBS Journal 273 (2006), 13231329.

[5] Y. Sun, K. Jin, X.O. Mao, Y. Zhu and D.A. Greenberg, PNAS 98 (2001), 15306-15311.

[6] Y. Sun, K. Jin, A. Peel, X.O. Mao, L. Xie and D.A. Greenberg, PNAS 100 (2007), 3497-3500.

[7] S. Herold, A. Fago, R.E. Weber, S. Dewilde and L. Moens, The Journal of Biological Chemistry 279 (2004), $22841-22847$.

[8] E. Fordel, L. Thijs, W. Martinet, D. Schrijvers, L. Moens and S. Dewilde, Gene 398 (2007), 114-122.

[9] F. Trandafir, D. Hoogewijs, F. Altieri, P. Rivetti di Val Cervo, K. Ramser, S. Van Doorslaer, J. Vanfleteren, L. Moens and S. Dewilde, Gene 398 (2007), 103-113.

[10] D. Peroni, A. Negro, M. Bähr and G.P.H. Dietz, Neuroscience Letters 421 (2007), 110-114.

[11] E. Vinck, S. Van Doorslaer, S. Dewilde and L. Moens, Journal of the American Chemical Society 126 (2004), $4516-4517$.

[12] H. Brunner and H. Sussner, Biochimica et Biophysica Acta 310 (1973), 20-31.

[13] T.G. Spiro and T.C. Strekas, Journal of the American Chemical Society 96 (1974), 338-345.

[14] T. Egawa and S.-R. Yeh, Journal of Inorganic Biochemistry 99 (2005), 72-96.

[15] D.J. Beebe, G.A. Mensing and G.M. Walker, Annual Review of Biomedical Engineering 4 (2002), 261-286.

[16] M.J. Lang and S. Block, American Journal of Physics 71 (2003), 201-212.

[17] E. Eriksson, J. Enger, B. Nordlander, N. Erjavec, K. Ramser, M. Goksör, S. Hohmann, T. Nyström and D. Hanstorp, Lab on a Chip 7 (2007), 71.

[18] K. Ramser, K. Logg, J. Enger, M. Goksör, M. Käll and D. Hanstorp, in: Proceedings of SPIE, Vol. 5514, Denver, 2004, pp. 560-568.

[19] K. Ramser, W. Wenseleers, S. Dewilde, S. Van Doorslaer, L. Moens and D. Hanstorp, Journal of Biomedical Optics 12 (2007), 044009-1-044009-9.

[20] K. Ramser, W. Wenseleers, S. Dewilde, S. Van Doorslaer, L. Moens and D. Hanstorp, Journal of Biochemical and Biophysical Methods 70 (2007), 627-633.

[21] K. Ramser, J. Enger, M. Goksör, D. Hanstorp, K. Logg and M. Käll, Lab on a Chip 5 (2004), 431-436.

[22] S. Dewilde, L. Kiger, T. Burmester, T. Hankeln, V. Baudin-Creuza, T. Aerts, M. Marden, R. Caubergs and L. Moens, Journal of Biological Chemistry 276 (2001), 38949-38955.

[23] M. Gouterman, The Porphyrins, Academic Press, New York, 1978.

[24] K. Ramser, E.J. Bjerneld, C. Fant and M. Käll, Journal of Biomedical Optics 8 (2003), 173-178.

[25] G.J. Puppels, J.H.F. Olminkhof, G.M.J. Segers-Nolten, C. Otto, F.F.M. DeMul and J. Greve, Experimental Cell Research 195 (1991), 361-367.

[26] B.R. Wood and D. McNaughton, Journal of Raman Spectroscopy 33 (2002), 517-523.

[27] K. König, H. Schneckenburger, R.A. and R. Steiner, J. Photochem. Photobiol. B: Biol. 18 (1993), 287-290.

[28] R. Rotomskis, G. Streckyte and S. Bagdonas, J. Photochem. Photobiol. B: Biol. 39 (1997), 172-175. 


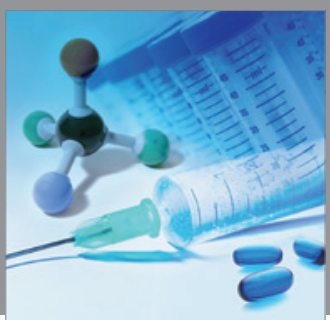

International Journal of

Medicinal Chemistry

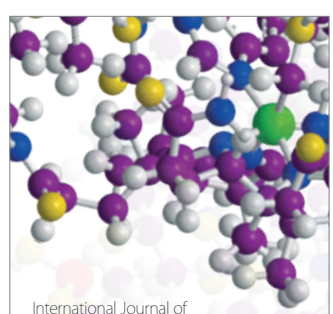

Carbohydrate Chemistry

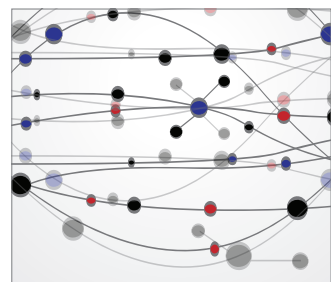

The Scientific World Journal
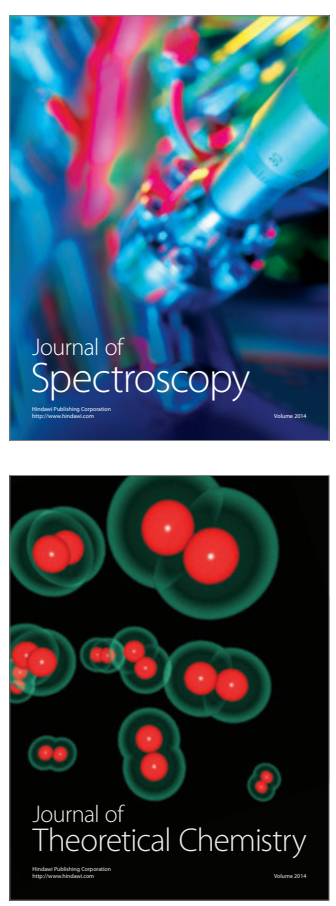
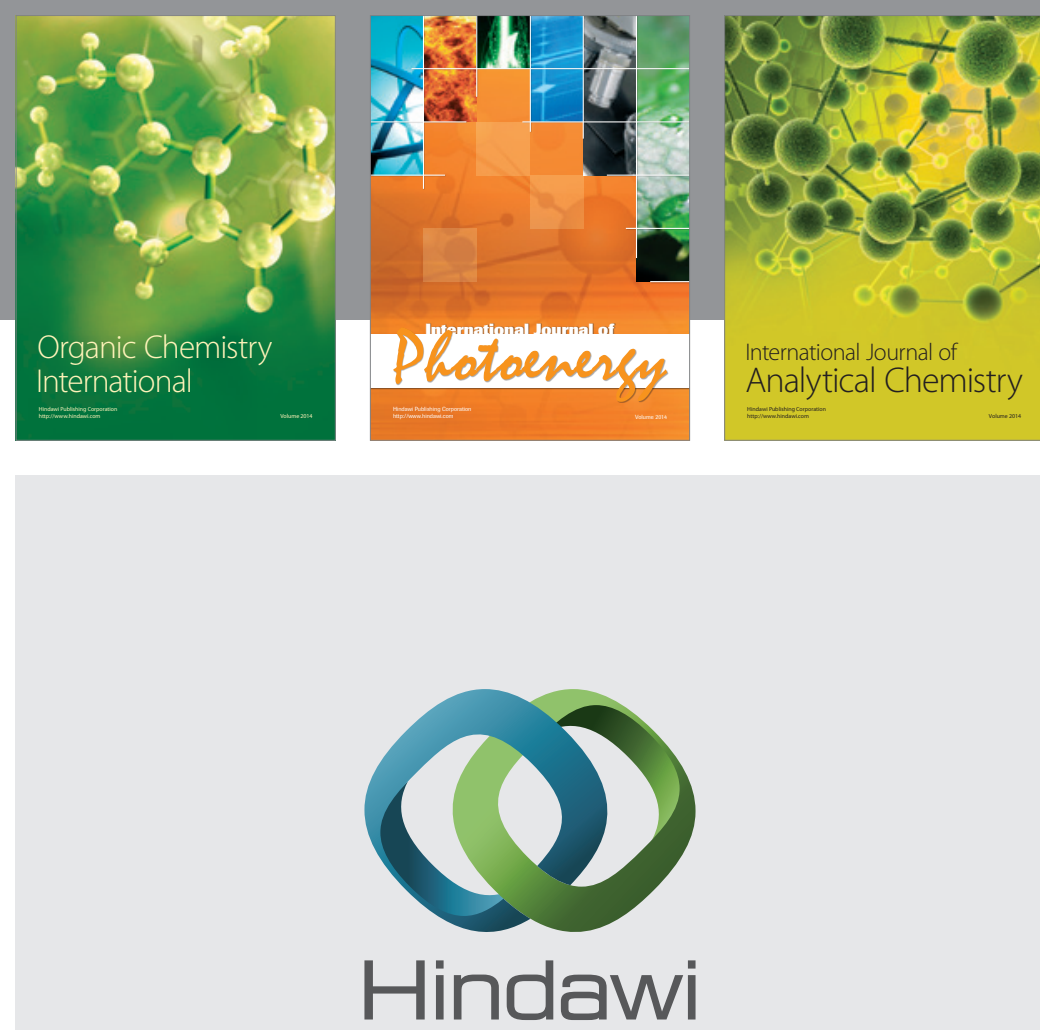

Submit your manuscripts at

http://www.hindawi.com
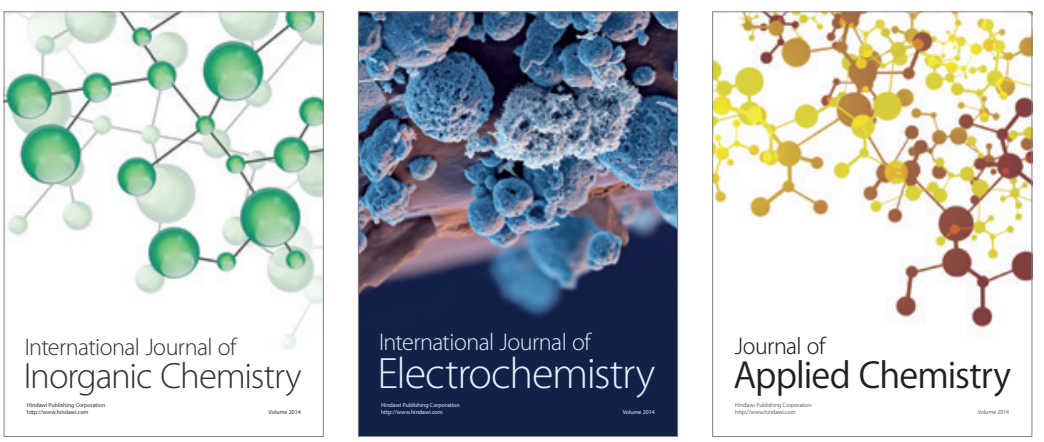

Journal of

Applied Chemistry
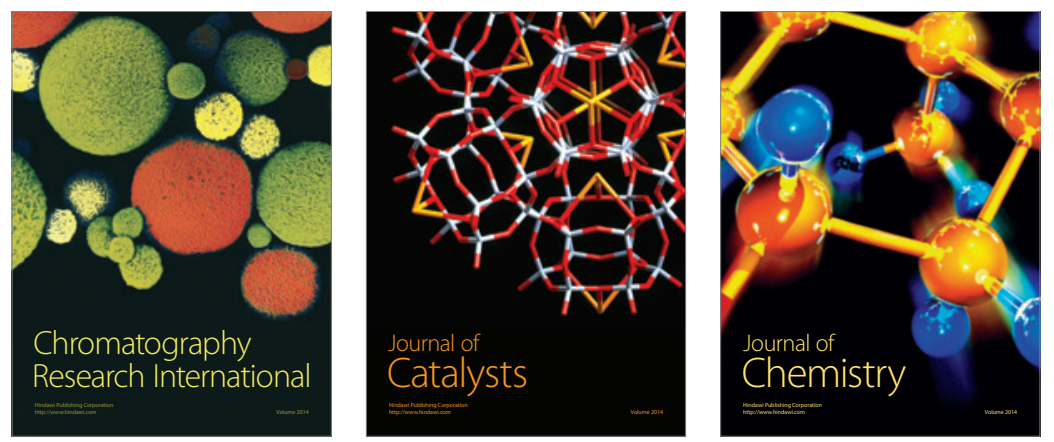
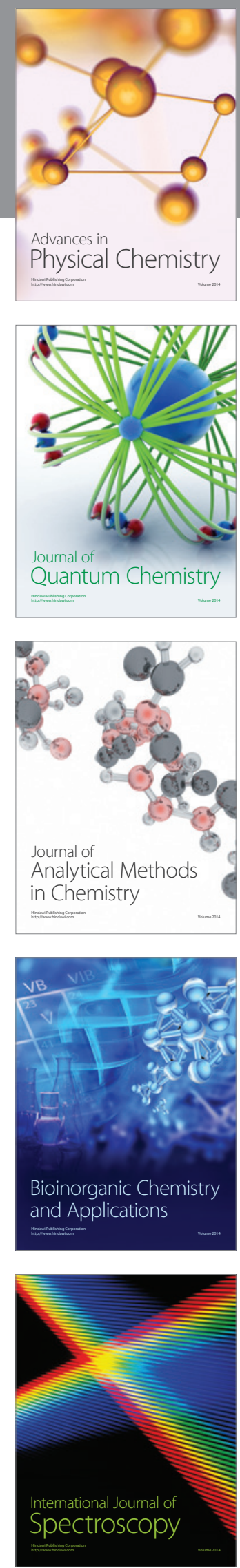